\title{
Failure Analysis of Brass Components
}

\author{
Chirag G. Shah
}

Exova Inc, 194 International Blvd, Glendale Heights, IL 60139

Brass components are used in a wide variety of applications, from hydraulic valves and fittings, plumbing and marine hardware to architecture and fasteners. Principal failure modes analyzed using the optical / electron microscopy and metallographic techniques that lead to in-service failures of brass components will be presented. Case histories involving stress corrosion cracking, dealloying (dezincification), fatigue and overstress failures of brass components will be discussed.

Brass is a metal alloy of Copper and Zinc, with copper content ranging from $58 \%$ to $95 \%$. In addition to Zinc, the major alloying element, small additions (less than 5\%) of other alloying elements are added to modify the properties so that the resulting material is fit for a given purpose. Brasses containing a minimum of $63 \%$ copper are termed alpha brasses or cold working Brasses. Brasses containing 35\%-45\% zinc are known as alphabeta or duplex brasses because they contain a mixture of the original solid solution (alpha phase) and a new solid solution of higher zinc content (beta phase). There are over 40 standard compositions of Brass.

Stress corrosion cracking (SCC or "season cracking") occurs only in the simultaneous presence of a sufficiently high tensile stress and a specific corrosive environment. For brasses the environment involved is usually reported to be involving ammonia or closely related substances such as amines. In dezincification, a porous layer of zinc free material is formed locally on the surface, leaving behind a porous, copper-rich structure that has inferior mechanical properties. Dezincification in the high-zinc alloys can occur in a wide variety of acid, neutral and alkaline media. The factors affecting the SCC and Dezincification of brass alloys will be discussed.

We will also discuss the overstress and fatigue failure of various brass components. Optical and electron microscope images of the various failed brass alloys will be presented. 


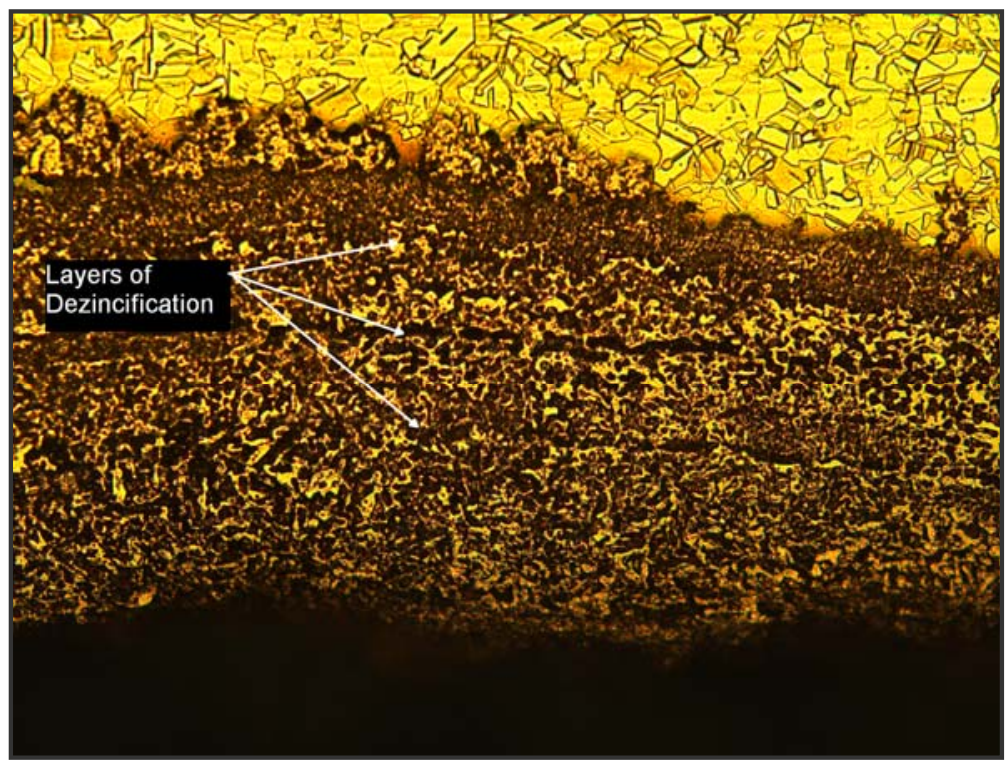

Figure 1: This optical micrograph shows a Dezinicified area of brass contact indicating a layered microstructure consistent with dezincification occurring over time.

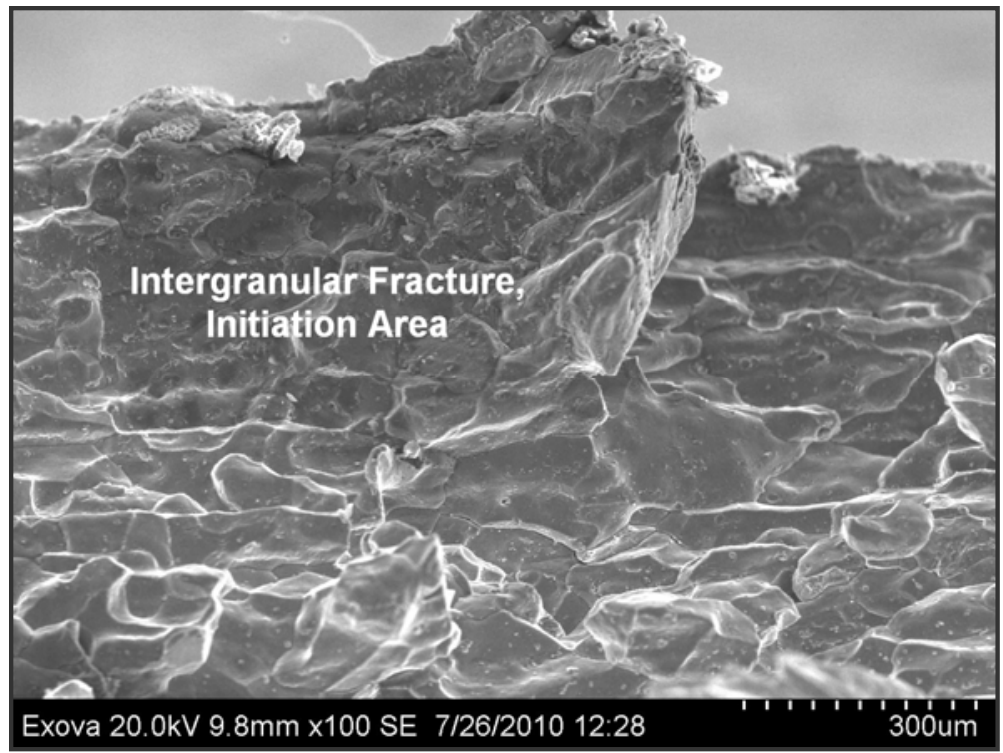

Figure 2: This secondary electron SEM image shows the intergranular fracture associated with the stress corrosion cracked brass fitting. 